\title{
Riesz Kernels and Pseudodifferential Operators Attached to Quadratic Forms Over $p$-adic Fields
}

\author{
O. Casas-Sánchez and W. A. Zúñiga-Galindo
}

\begin{abstract}
We study pseudodifferential equations and Riesz kernels attached to certain quadratic forms over $p$-adic fields. We attach to an elliptic quadratic form of dimension two or four a family of distributions depending on a complex parameter, the Riesz kernels, and show that these distributions form an Abelian group under convolution. This result implies the existence of fundamental solutions for certain pseudodifferential equations like in the classical case.
\end{abstract}

\section{Introduction}

This paper aims to study Riesz kernels and pseudodifferential equations attached to quadratic forms over $p$-adic fields. The Riesz kernels are naturally connected with several types of (pseudo) differential equations in the Archimedean setting, see e.g. [3], 9], 11], 12], and non Archimedean one, see e.g. [1], 8], 17, [18], [19, 21]. In particular, in the non Archimedean setting, Riesz kernels attach to 'polynomials of degree one' has been used to solve pseudodifferential equations [1, 8], 21. Our initial motivation was to extend these results to the case of polynomials of higher degree to obtain $p$-adic analogs of the results of $[\mathbf{3}, \mathbf{1 2}$. To present our results consider the diagonal quadratic form $f(\xi)=a_{1} \xi_{1}^{2}+\ldots+a_{n} \xi_{n}^{2}$, the local zeta function attached to $f$ is the distribution defined by

$$
\left(|f|_{p}^{s}, \phi\right)=\int_{\mathbb{Q}_{p}^{n} \backslash f^{-1}(0)}|f(\xi)|_{p}^{s} \phi(\xi) d^{n} \xi, \operatorname{Re}(s)>0 .
$$

These distributions, called local zeta functions, were introduced in the 50 's by I. Gel'fand and A. Weil, see [4, 5. A Riesz kernel is a local zeta function multiplied by a suitable gamma factor. In the cases in which $n=2,4$ and the quadratic form is elliptic, we show that the Riesz kernels, considered as distributions on certain $p$-adic Lizorkin spaces, form an Abelian group under the operation of convolution, see Theorem 2 and Remark 2. The proof of this fact depends on a theorem of Rallis-Schiffmann that asserts that the distributions of type $|f|_{p}^{s}$ satisfy certain functional equations, see [10, also [7, [13, 14, [15. In order to use this result

2000 Mathematics Subject Classification. Primary 35S05, 11S40; Secondary $26 \mathrm{E} 30$.

Key words and phrases. Pseudodifferential equations, Riesz kernels, Local zeta functions, Non-Archimedean analysis.

The second author was partially supported by CONACYT under Grant \# 127794 . 
we compute all the gamma factors that appear in the functional equation for $p$-adic quadratic forms of type $a_{1} \xi_{1}^{2}+a_{2} \xi_{2}^{2}+\cdots+a_{n} \xi_{n}^{2}$, see Theorem 1 As consequence, we obtain fundamental solutions for certain pseudodifferential equations, see Theorem 3 an Remark 3. The fundamental solutions presented here are 'classical solutions', see Definition 3, while those present in [8] and [24-[26] are 'weak solutions'. We also obtain the existence of a pseudodifferential operator $\boldsymbol{f}(\partial, 1)$, acting on a space of Lizorkin distributions, and a gamma factor $A(s)$ such that $\boldsymbol{f}(\partial, 1)|f|_{p}^{s+1}=$ $A(s)|f|_{p}^{s}$, where $f$ is an elliptic quadratic form of dimension 2 or 4 , see Theorem 3 and Remark 3. This is a non Archimedean pseudodifferential (particular) version of a celebrated result of Sato-Bernstein, see [13, [5], 26. Section 6.1.2]. Thus, a natural problem is to study the existence of pseudodifferential Sato-Bernstein operators, and the corresponding functions, in the setting $p$-adic prehomogeneous vector spaces, this problem was posed in [26. Section 6.1.2]. Finally, the results obtained here can be applied to study other types of pseudodifferential equations, these results will appear in a separate article elsewhere.

Acknowledgement. The authors want to thank to Professor Fumihiro Sato for his kind assistance on the functional equation for the local zeta function attached to a quadratic form. In particular, we are very grateful to him for allowing us to use some of the ideas of his unpublished manuscript [16. The second author wants to thank to Professor Sergii Torba for several useful comments and discussions about this article. The authors also want to thank to the referee for his/her careful reading of the article.

\section{Preliminaries}

In this section we fix the notation and collect some basic results on $p$-adic analysis that we will use through the article. For a detailed exposition on $p$-adic analysis the reader may consult [1], [19], [21].

2.1. The field of $p$-adic numbers. Along this article $p$ will denote a prime number different from 2 . The field of $p$-adic numbers $\mathbb{Q}_{p}$ is defined as the completion of the field of rational numbers $\mathbb{Q}$ with respect to the $p$-adic norm $|\cdot|_{p}$, which is defined as

$$
|x|_{p}= \begin{cases}0 & \text { if } x=0 \\ p^{-\gamma} & \text { if } x=p^{\gamma} \frac{a}{b},\end{cases}
$$

where $a$ and $b$ are integers coprime with $p$. The integer $\gamma:=\operatorname{ord}(x)$, with $\operatorname{ord}(0):=$ $+\infty$, is called the $p$-adic order of $x$. We extend the $p$-adic norm to $\mathbb{Q}_{p}^{n}$ by taking

$$
\|x\|_{p}:=\max _{1 \leq i \leq n}\left|x_{i}\right|_{p}, \quad \text { for } x=\left(x_{1}, \ldots, x_{n}\right) \in \mathbb{Q}_{p}^{n} .
$$

We define $\operatorname{ord}(x)=\min _{1 \leq i \leq n}\left\{\operatorname{ord}\left(x_{i}\right)\right\}$, then $\|x\|_{p}=p^{-\operatorname{ord}(x)}$. Any $p$-adic number $x \neq 0$ has a unique expansion $x=p^{\operatorname{ord}(x)} \sum_{j=0}^{\infty} x_{i} p^{j}$, where $x_{j} \in\{0,1,2, \ldots, p-1\}$ and $x_{0} \neq 0$. By using this expansion, we define the fractional part of $x \in \mathbb{Q}_{p}$, denoted $\{x\}_{p}$, as the rational number

$$
\{x\}_{p}= \begin{cases}0 & \text { if } x=0 \text { or } \operatorname{ord}(x) \geq 0 \\ p^{\operatorname{ord}(x)} \sum_{j=0}^{-\operatorname{ord}(x)-1} x_{j} p^{j} & \text { if } \operatorname{ord}(x)<0 .\end{cases}
$$

For $\gamma \in \mathbb{Z}$, denote by $B_{\gamma}^{n}(a)=\left\{x \in \mathbb{Q}_{p}^{n}:\|x-a\|_{p} \leq p^{\gamma}\right\}$ the ball of radius $p^{\gamma}$ with center at $a=\left(a_{1}, \ldots, a_{n}\right) \in \mathbb{Q}_{p}^{n}$, and take $B_{\gamma}^{n}(0):=B_{\gamma}^{n}$. Note that 
$B_{\gamma}^{n}(a)=B_{\gamma}\left(a_{1}\right) \times \cdots \times B_{\gamma}\left(a_{n}\right)$, where $B_{\gamma}\left(a_{i}\right):=\left\{x \in \mathbb{Q}_{p}:\left|x_{i}-a_{i}\right|_{p} \leq p^{\gamma}\right\}$ is the one-dimensional ball of radius $p^{\gamma}$ with center at $a_{i} \in \mathbb{Q}_{p}$. The ball $B_{0}^{n}(0)$ is equals the product of $n$ copies of $B_{0}(0):=\mathbb{Z}_{p}$, the ring of $p$-adic integers.

2.2. The Bruhat-Schwartz space. A complex-valued function $\varphi$ defined on $\mathbb{Q}_{p}^{n}$ is called locally constant if for any $x \in \mathbb{Q}_{p}^{n}$ there exist an integer $l(x) \in \mathbb{Z}$ such that

$$
\varphi\left(x+x^{\prime}\right)=\varphi(x) \text { for } x^{\prime} \in B_{l(x)}^{n} .
$$

A function $\varphi: \mathbb{Q}_{p}^{n} \rightarrow \mathbb{C}$ is called a Bruhat-Schwartz function (or a test function) if it is locally constant with compact support. The $\mathbb{C}$-vector space of Bruhat-Schwartz functions is denoted by $\mathbf{S}\left(\mathbb{Q}_{p}^{n}\right)$. For $\varphi \in \mathbf{S}\left(\mathbb{Q}_{p}^{n}\right)$, the largest of such number $l=l(\varphi)$ satisfying (2.1) is called the exponent of local constancy of $\varphi$.

Let $\mathbf{S}^{\prime}\left(\mathbb{Q}_{p}^{n}\right)$ denote the set of all functionals (distributions) on $\mathbf{S}\left(\mathbb{Q}_{p}^{n}\right)$. All functionals on $\mathbf{S}\left(\mathbb{Q}_{p}^{n}\right)$ are continuous.

Set $\chi(y)=\exp \left(2 \pi i\{y\}_{p}\right)$ for $y \in \mathbb{Q}_{p}$. The map $\chi(\cdot)$ is an additive character on $\mathbb{Q}_{p}$, i.e. a continuos map from $\mathbb{Q}_{p}$ into $S$ (the unit circle) satisfying $\chi\left(y_{0}+y_{1}\right)=$ $\chi\left(y_{0}\right) \chi\left(y_{1}\right), y_{0}, y_{1} \in \mathbb{Q}_{p}$.

Given $\xi=\left(\xi_{1}, \ldots, \xi_{n}\right)$ and $x=\left(x_{1}, \ldots, x_{n}\right) \in \mathbb{Q}_{p}^{n}$, we set $\xi \cdot x:=\sum_{j=1}^{n} \xi_{j} x_{j}$. The Fourier transform of $\varphi \in \mathbf{S}\left(\mathbb{Q}_{p}^{n}\right)$ is defined as

$$
(\mathcal{F} \varphi)(\xi)=\int_{\mathbb{Q}_{p}^{n}} \chi(-\xi \cdot x) \varphi(\xi) d^{n} x \quad \text { for } \xi \in \mathbb{Q}_{p}^{n},
$$

where $d^{n} x$ is the Haar measure on $\mathbb{Q}_{p}^{n}$ normalized by the condition $\operatorname{vol}\left(B_{0}^{n}\right)=1$. The Fourier transform is a linear isomorphism from $\mathbf{S}\left(\mathbb{Q}_{p}^{n}\right)$ onto itself satisfying $(\mathcal{F}(\mathcal{F} \varphi))(\xi)=\varphi(-\xi)$. We will also use the notation $\mathcal{F}_{x \rightarrow \xi} \varphi$ and $\widehat{\varphi}$ for the Fourier transform of $\varphi$.

2.3. Operations on Distributions. Let $\Omega$ denote the characteristic function of the interval $[0,1]$. Then $\Delta_{k}(x):=\Omega\left(p^{-k}\|x\|_{p}\right)$ is the characteristic function of the ball $B_{k}^{n}(0)$.

2.3.1. Convolution. Given $f, g \in \mathbf{S}^{\prime}\left(\mathbb{Q}_{p}^{n}\right)$, their convolution $f * g$ is defined by

$$
\langle f * g, \varphi\rangle=\lim _{k \rightarrow+\infty}\left\langle f(y) \times g(x), \Delta_{k}(x) \varphi(x+y)\right\rangle
$$

if the limit exists for all $\varphi \in \mathbf{S}\left(\mathbb{Q}_{p}^{n}\right)$. We recall that if $f * g$ exists, then $g * f$ exists and $f * g=g * f$, see e.g. [21, Section VII.1]. In the case in which $g=\psi \in \mathbf{S}\left(\mathbb{Q}_{p}^{n}\right)$,

$$
f * \psi(x)=\langle f(y), \psi(x-y)\rangle,
$$

see e.g. 21, Section VII.1].

2.3.2. Fourier transform. The Fourier transform $\mathcal{F}[f]$ of a distribution $f \in$ $\mathbf{S}^{\prime}\left(\mathbb{Q}_{p}^{n}\right)$ is defined by

$$
\langle\mathcal{F}[f], \varphi\rangle=\langle f, \mathcal{F}[\varphi]\rangle \text { for all } \varphi \in \mathbf{S}\left(\mathbb{Q}_{p}^{n}\right) .
$$

The Fourier transform $f \rightarrow \mathcal{F}[f]$ is a linear isomorphism from $\mathbf{S}^{\prime}\left(\mathbb{Q}_{p}^{n}\right)$ onto $\mathbf{S}^{\prime}\left(\mathbb{Q}_{p}^{n}\right)$. Furthermore, $f=\mathcal{F}[\mathcal{F}[f](-\xi)]$. 
2.3.3. Multiplication. Set $\delta_{k}(x):=p^{n k} \Omega\left(p^{k}\|x\|_{p}\right)$ for $k \in \mathbb{N}$. Given $f, g \in$ $\mathbf{S}^{\prime}\left(\mathbb{Q}_{p}^{n}\right)$, their product $f \cdot g$ is defined by

$$
\langle f \cdot g, \varphi\rangle=\lim _{k \rightarrow+\infty}\left\langle g,\left(f * \delta_{k}\right) \varphi\right\rangle
$$

if the limit exists for all $\varphi \in \mathbf{S}\left(\mathbb{Q}_{p}^{n}\right)$. We recall that the existence of the product $f \cdot g$ is equivalent to the existence of $\mathcal{F}[f] * \mathcal{F}[g]$. In addition, $\mathcal{F}[f \cdot g]=\mathcal{F}[f] * \mathcal{F}[g]$ and $\mathcal{F}[f * g]=\mathcal{F}[f] \cdot \mathcal{F}[g]$, see e.g. [21, Section VII.5]. The following result will be used later on.

LEMma 1 ([21, Section VII.5].). Let $f, g$ functions in $L_{l o c}^{1}$ for which the function

$$
\int_{\mathbb{Q}_{p}^{n}} g(x) \varphi(x) f(x-\xi) d^{n} x
$$

is continuos at $\xi=0 \in \mathbb{Q}_{p}^{n}$, for any $\varphi \in \mathbf{S}\left(\mathbb{Q}_{p}^{n}\right)$. Then the product $f \cdot g$ is in $\mathbf{S}^{\prime}\left(\mathbb{Q}_{p}^{n}\right)$ and the distribution is induced by the pointwise product $f(x) g(x)$. by

2.4. The Hilbert Symbol. The Hilbert symbol $(a, b)_{p}, a, b \in \mathbb{Q}_{p}^{\times}$, is defined $(a, b)_{p}= \begin{cases}1 & \text { if } a x^{2}+b y^{2}-z^{2}=0 \text { has a solution }(x, y, z) \neq(0,0,0) \text { in } \mathbb{Q}_{p}^{3} \\ -1 & \text { otherwise. }\end{cases}$

The Hilbert symbol possesses the following properties (see e.g. Theorem 3.3.1 [6]):

$$
\begin{gathered}
(a, b)_{p}=(b, a)_{p} \text { and }\left(a, c^{2}\right)_{p}=1, \text { for } a, b, c \in \mathbb{Q}_{p}^{\times} ; \\
(a b, c)_{p}=(a, c)_{p}(b, c)_{p}, \text { for } a, b, c \in \mathbb{Q}_{p}^{\times} ; \\
\begin{cases}(a, b)_{p}=1 & \text { for } a, b \in \mathbb{Z}_{p}^{\times} \\
(a, p)_{p}=\left(\frac{a_{0}}{p}\right) & \text { for } a \in \mathbb{Z}_{p}^{\times},\end{cases}
\end{gathered}
$$

where $a_{0} \in \mathbb{Z}$, with $a \equiv a_{0} \bmod \mathbb{Z}_{p}$, and $\left(\frac{a_{0}}{p}\right)$ is the Legendre symbol.

Along this article $\left[\mathbb{Q}_{p}^{\times}\right]^{2}$ denotes the subgroup of squares of $\mathbb{Q}_{p}^{\times}$. We recall that $\mathbb{Q}_{p}^{\times} /\left[\mathbb{Q}_{p}^{\times}\right]^{2}$ is a finite group with four elements. We fix $\{1, \epsilon, p, \epsilon p\}$ to be a set of representatives, here $\epsilon$ is unit which is not square.

It is clear that $(a, b)_{p}$ does not change when $a$ and $b$ are multiplied by squares, thus the Hilbert symbol gives rise a map from $\mathbb{Q}_{p}^{\times} /\left[\mathbb{Q}_{p}^{\times}\right]^{2} \times \mathbb{Q}_{p}^{\times} /\left[\mathbb{Q}_{p}^{\times}\right]^{2}$ into $\{1,-1\}$. For a fixed $\beta \in \mathbb{Q}_{p}^{\times}, \pi_{\beta}(t)=(\beta, t)_{p}$ defines a multiplicative character on $\mathbb{Q}_{p}^{\times}$, the multiplicatively of $\pi_{\beta}$ follows from property (2.3). 


\subsection{The Weil Constant. Let}

$$
f(x):=a_{1} x_{1}^{2}+a_{2} x_{2}^{2}+\cdots+a_{n} x_{n}^{2}, \quad a_{i} \in \mathbb{Q}_{p}^{\times}, \quad i=1,2, \ldots, n,
$$

be a quadratic form. A such quadratic form is characterized by three invariants:

(i) the dimension $n$;

(ii) the discriminant $D=a_{1} a_{2} \cdots a_{n} \bmod \left[\mathbb{Q}_{p}^{\times}\right]^{2}$;

(iii) the Hasse invariant $H=\prod_{i<j}\left(a_{i}, a_{j}\right)_{p}$.

By [22, Theoreme 2], see also [10, Theoreme 1.1], there exist a complex constant $\gamma(f)$ of absolute value one, such that

$$
\begin{aligned}
& \int_{\mathbb{Q}_{p}^{n}} \hat{\varphi}(x) \chi(t f(x)) d^{n} x \\
& =\gamma(t f)|t|_{p}^{-n / 2}|D|_{p}^{-1 / 2} \int_{\mathbb{Q}_{p}^{n}} \varphi(x) \chi\left(-\frac{1}{t} f\left(\frac{x_{1}}{2 a_{1}}, \ldots, \frac{x_{n}}{2 a_{n}}\right)\right) d^{n} x,
\end{aligned}
$$

for all $t \in \mathbb{Q}_{p}^{\times}$, where $D=a_{1} a_{2} \cdots a_{n}$.

Since $\gamma(f)=\gamma\left(a_{1} x_{1}^{2}\right) \cdots \gamma\left(a_{n} x_{n}^{2}\right)$, see e.g. [22, p. 173], the calculation of $\gamma(f)$ is reduced to the case $n=1$. For a $\alpha \in \mathbb{Q}_{p}^{\times}$, we set $\gamma(\alpha):=\gamma\left(\alpha x_{1}^{2}\right)$.

Lemma 2. For a unit $u \in \mathbb{Z}_{p}^{\times}$, with $u \equiv u_{0} \bmod p \mathbb{Z}_{p}$, we have $\gamma(u)=1$ and $\gamma(u p)=\left(\frac{u_{0}}{p}\right) \sigma_{p}$, where

$$
\sigma_{p}:= \begin{cases}1 & \text { if } p \equiv 1 \bmod 4 \\ \sqrt{-1} & \text { if } p \equiv 3 \bmod 4\end{cases}
$$

Proof. Take $\varphi(x)$ to be the characteristic function of $\mathbb{Z}_{p}$ and $u \in \mathbb{Z}_{p}^{\times}$, by (2.6)

$$
\begin{aligned}
\int_{\mathbb{Q}_{p}} \hat{\varphi}(x) \chi\left(u x^{2}\right) d x & =\gamma(u)|u|_{p}^{-1 / 2} \int_{\mathbb{Q}_{p}} \varphi(x) \chi\left(-\frac{x^{2}}{4 u}\right) d x \\
\int_{\mathbb{Z}_{p}} d x & =\gamma(u) .
\end{aligned}
$$

In the case up with $u \in \mathbb{Z}_{p}^{\times}$, by applying (2.6) we have

$$
\begin{aligned}
\int_{\mathbb{Q}_{p}} \hat{\varphi}(x) \chi\left(u p x^{2}\right) d x & =\gamma(u p)|u p|_{p}^{-1 / 2} \int_{\mathbb{Q}_{p}} \varphi(x) \chi\left(-\frac{x^{2}}{4 p u}\right) d x \\
\int_{\mathbb{Z}_{p}} \chi\left(u p x^{2}\right) d x & =\gamma(u p) p^{1 / 2} \int_{\mathbb{Z}_{p}} \chi\left(-\frac{x^{2}}{4 p u}\right) d x \\
1 & =\gamma(u p) p^{1 / 2} \int_{\mathbb{Z}_{p}} \chi\left(-\frac{x^{2}}{4 p u}\right) d x .
\end{aligned}
$$


If $z \in \mathbb{Z}_{p} \backslash\{0\}$ we set $z=z_{0}+z_{1} p+\ldots+z_{k} p^{k}+\ldots$ with $z_{k}=\{0,1, \ldots, p-1\}$. Now by changing variables $(x=2 u y)$ in the previous integral:

$$
\begin{aligned}
\int_{\mathbb{Z}_{p}} \chi\left(-\frac{x^{2}}{4 p u}\right) d x & =\int_{|y|_{p} \leq 1} \chi\left(-\frac{u y^{2}}{p}\right) d y \\
& =\int_{|y|_{p}=1} \chi\left(-\frac{u y^{2}}{p}\right) d y+\int_{|y|_{p}<1} \chi\left(-\frac{u y^{2}}{p}\right) d y \\
& =\frac{1}{p} \sum_{y_{0}=1}^{p-1} \exp \left\{-2 \pi i \frac{u_{0} y_{0}^{2}}{p}\right\}+\frac{1}{p}=\frac{1}{p} \sum_{y_{0}=0}^{p-1} \exp \left\{-2 \pi i \frac{u_{0} y_{0}^{2}}{p}\right\} \\
& =p^{-1 / 2}\left(\frac{-u_{0}}{p}\right) \sigma_{p},
\end{aligned}
$$

where in the last step we used a result of Gauss on quadratic exponential sums, see e.g. [21, p. 55]. Therefore

$$
\gamma(u p)=\frac{1}{\left(\frac{-1}{p}\right)\left(\frac{u_{0}}{p}\right) \sigma_{p}}=\left(\frac{u_{0}}{p}\right) \sigma_{p} .
$$

The next lemma shows the relation between the constant $\gamma$ and the Hilbert symbol.

LEMMA 3. With the above notation, the following assertions hold.

(i) $\gamma(-a) \gamma(a)=1$.

(ii) Set $h(x)=x_{1}^{2}-a x_{2}^{2}-b x_{3}^{2}+a b x_{4}^{2}$ with $a, b \in \mathbb{Q}_{p}^{\times}$. Then

$$
\gamma(h)=\gamma(1) \gamma(-a) \gamma(-b) \gamma(a b)=(a, b)_{p} .
$$

(iii) If $n \equiv 0 \bmod 2$, then $\gamma(t f)=\gamma(f)\left(t, D^{*}\right)_{p}$ for any $t \in \mathbb{Q}_{p}^{\times}$, where

$$
D^{*}:=(-1)^{\frac{n}{2}} D \text {. }
$$

Proof. (i) See [22, Section No. 25, p. 173 ]. (ii) See [22, Section No. 28, p. 176]. (iii) See [10, Proposition 1.7].

2.6. Local zeta functions. For $a>0$ and $s \in \mathbb{C}$ we set $a^{s}:=e^{s \ln a}$. Let $f(x)$ be a quadratic form over $\mathbb{Q}_{p}$ and $\pi_{\beta}(t):=(\beta, t)_{p}, \quad t \in \mathbb{Q}_{p}^{\times}$as before. The local function zeta attached to $\left(f, \pi_{\beta}\right)$ is the distribution given by

$$
Z_{\varphi}\left(s, \pi_{\beta}, f\right):=Z_{\varphi}\left(s, \pi_{\beta}\right)=\int_{\mathbb{Q}_{p}^{n} \backslash f^{-1}(0)} \pi_{\beta}(f(x))|f(x)|_{p}^{s-n / 2} \varphi(x) d^{n} x,
$$

$\varphi \in \mathbf{S}\left(\mathbb{Q}_{p}^{n}\right)$ and $\operatorname{Re}(s)>\frac{n}{2}$. If $\beta=1$ we use $Z_{\varphi}(s, f)$ instead of $Z_{\varphi}\left(s, \pi_{1}, f\right)$. The local zeta functions are defined for arbitrary polynomials and arbitrary multiplicative characters. These objects were introduced in the 60 's by A. Weil and since then they have been studied intensively, see e.g. [5. The local zeta function $Z_{\varphi}\left(s, \pi_{\beta}\right)$ is a distribution on $\mathbf{S}\left(\mathbb{Q}_{p}^{n}\right)$ for $\operatorname{Re}(s)>\frac{n}{2}$, which admits a meromorphic continuation to the whole complex plane (for arbitrary $f$ and $\pi_{\beta}$ ) such that $Z_{\varphi}\left(s, \pi_{\beta}\right)$ is a rational function of $p^{-s}$, see [5, Theorem 8.2.1]. 
2.7. Functional equations. It is well-known that the Fourier transform of the distribution $\pi_{\beta}(t)|t|_{p}^{s-1}$ is $\rho\left(\pi_{\beta}, s\right) \pi_{\beta}^{-1}(t)|t|_{p}^{-s}$ i.e.

$$
\int_{\mathbb{Q}_{p}^{\times}} \widehat{\varphi}(t) \pi_{\beta}(t)|t|_{p}^{s-1} d t=\rho\left(\pi_{\beta}, s\right) \int_{\mathbb{Q}_{p}^{\times}} \varphi(t) \pi_{\beta}^{-1}(t)|t|_{p}^{-s} d t,
$$

for all $\varphi(t) \in S\left(\mathbb{Q}_{p}\right)$, see e.g. 21, Section VIII.2]. We recall that (2.9) is a particular case of the functional equation for the Iwasawa-Tate local zeta function see e.g. 20 Theoerem 2.4.1 and Lemma 2.4.3].

We now compute the factors $\rho\left(\pi_{\beta}, s\right)$ appearing in (2.9).

Lemma 4. Set $\mathbb{Q}_{p}^{\times} /\left[\mathbb{Q}_{p}^{\times}\right]^{2}=\{1, \epsilon, p, \epsilon p\}$ where $\epsilon$ is unit which is not square. Then

(i) $\rho\left(\pi_{1}, s\right)=\frac{1-p^{s-1}}{1-p^{-s}}$;

(ii) $\rho\left(\pi_{\epsilon}, s\right)=\frac{1+p^{s-1}}{1+p^{-s}}$;

(iii) $\rho\left(\pi_{\eta}, s\right)= \pm \sigma_{p} p^{s-\frac{1}{2}}, \eta=p, \epsilon p$ with $\sigma_{p}$ as in (2.7).

Proof. (i) Take $\varphi(t)$ to be the characteristic function of $\mathbb{Z}_{p}$ in (2.9), then

$$
\rho\left(\pi_{1}, s\right)=\frac{\int_{\mathbb{Z}_{p} \backslash\{0\}}|t|_{p}^{s-1} d t}{\int_{\mathbb{Z}_{p} \backslash\{0\}}|t|_{p}^{-s} d t}=\frac{1-p^{s-1}}{1-p^{-s}} .
$$

(ii) Note that $\pi_{\epsilon}(t)=(-1)^{\operatorname{ord}(t)}$, see [21, Lemma on p. 130], by taking $\varphi(t)$ to be the characteristic function of $\mathbb{Z}_{p}$ in (2.9), we have

$$
\rho\left(\pi_{\epsilon}, s\right)=\frac{1+p^{s-1}}{1+p^{-s}} \text {. }
$$

(iii) Set

$$
\mathbb{Q}_{p, \eta}^{\times}:=\left\{x \in \mathbb{Q}_{p}^{\times} \mid x=a^{2}-\eta b^{2}, \quad a, b \in \mathbb{Q}_{p}\right\}
$$

and

$$
\operatorname{sgn}_{\eta}(x):= \begin{cases}1 & \text { if } x \in \mathbb{Q}_{p, \eta}^{\times} \\ -1 & \text { if } x \notin \mathbb{Q}_{p, \eta}^{\times} .\end{cases}
$$

In [21, p. 129] is proved that $\rho\left(\pi_{\eta}, s\right)= \pm \sqrt{s g n_{\eta}(-1)} p^{s-\frac{1}{2}}$ for $\eta=p, \epsilon p$. Since $(t, \eta)_{p}=s g n_{\eta}(t)$ we have

$$
\pm \sqrt{\operatorname{sgn}_{\eta}(-1)}= \pm \sqrt{(\eta,-1)_{p}}= \pm \sqrt{\left(\frac{-1}{p}\right)}= \pm \sigma_{p} .
$$

Set $f^{*}(x):=f\left(\frac{x_{1}}{a_{1}}, \ldots, \frac{x_{n}}{a_{n}}\right)$, and

$$
Z_{\varphi}^{*}\left(s, \pi_{\beta}\right):=\int_{\mathbb{Q}_{p}^{n} \backslash f^{*-1}(0)} \pi_{\beta}\left(f^{*}(x)\right)\left|f^{*}(x)\right|_{p}^{s-n / 2} \varphi(x) d^{n} x .
$$

Theorem 1 ([10, Theoreme 22-13]). If $n \equiv 0 \bmod 2$, then $Z_{\varphi}(s)$ satisfies

$$
Z_{\hat{\varphi}}(s)=\rho\left(\pi_{1}, s-\frac{n}{2}+1\right) \rho\left(\pi_{D^{*}}, s\right)|D|_{p}^{-1 / 2} \gamma(f) Z_{\varphi}^{*}\left(-s+n / 2, \pi_{D^{*}}\right)
$$

for any $\varphi \in \mathbf{S}\left(\mathbb{Q}_{p}^{n}\right)$. 
Proof. The announced formula is a particular case of formula 2-20 in 10. We note that our functional equation equals up to a constant to the functional equation in [10, this is due fact that we used a different normalization for the Haar measure.

\subsection{Some explicit functional equations.}

Corollary 1. Let $f(x)$ be as before. Assume that $n \equiv 0 \bmod 2$ and that $D^{*}$ is a square. Then

$$
Z_{\hat{\varphi}}(s)=\rho\left(\pi_{1}, s-\frac{n}{2}+1\right) \rho\left(\pi_{1}, s\right)|D|_{p}^{-1 / 2} \gamma(f) Z_{\varphi}^{*}(-s+n / 2)
$$

for any $\varphi \in \mathbf{S}\left(\mathbb{Q}_{p}^{n}\right)$.

Proposition 1. If $f(x)=x_{1}^{2}-\eta x_{2}^{2}, \eta=\epsilon, p, p \epsilon$, then

$$
\begin{aligned}
& \int_{\mathbb{Q}_{p}^{2} \backslash\{0\}}|f(x)|_{p}^{s-1} \widehat{\varphi}(x) d^{2} x \\
= & \begin{cases}\frac{1-p^{2(s-1)}}{1-p^{-2 s}} \int_{\mathbb{Q}_{p}^{2} \backslash\{0\}}\left|\eta x_{1}^{2}-x_{2}^{2}\right|_{p}^{-s} \varphi(x) d^{2} x \quad \text { if } \eta=\epsilon \\
\frac{1-p^{s-1}}{1-p^{-s}} \int_{\mathbb{Q}_{p}^{2} \backslash\{0\}}\left|\eta x_{1}^{2}-x_{2}^{2}\right|_{p}^{-s} \varphi(x) d^{2} x \quad \text { if } \eta=p, p \epsilon .\end{cases}
\end{aligned}
$$

Proof. Since $D^{*}=-D=\eta$ and $\pi_{-D}\left(f^{*}(x)\right)=\left(\eta, x_{1}^{2}-\eta x_{2}^{2}\right)_{p}=1$. By Theorem 1 we have

$$
\begin{gathered}
Z_{\widehat{\varphi}}(s)=\rho\left(\pi_{1}, s\right) \rho\left(\pi_{-D}, s\right)|D|_{p}^{-1 / 2} \gamma(f) Z_{\varphi}^{*}\left(1-s, \pi_{1}\right) \\
=\rho\left(\pi_{1}, s\right) \rho\left(\pi_{-D}, s\right)|D|_{p}^{-1 / 2} \gamma(f)|\eta|_{p}^{s} \int_{\mathbb{Q}_{p}^{2} \backslash\{0\}}\left|\eta x_{1}^{2}-x_{2}^{2}\right|_{p}^{-s} \varphi(x) d x_{1} d x_{2} .
\end{gathered}
$$

The announced functional equations follow from the following calculations. (i) Take $\eta=\epsilon$, then $|\epsilon|_{p}^{-1 / 2}=|\epsilon|_{p}^{s}=1, \quad \gamma(f)=\gamma(1) \gamma(-\epsilon)=1$, see Lemma 2, and $\pi_{-D}(\epsilon)=(\epsilon, \epsilon)_{p}=1$, see (2.4). Furthermore $\rho\left(\pi_{1}, s\right)=\frac{1-p^{s-1}}{1-p^{-s}}, \rho\left(\pi_{\epsilon}, s\right)=\frac{1+p^{s-1}}{1+p^{-s}}$, see Lemma 4

(ii) Take $\eta=p, p \epsilon$, in this case we have $|\eta|_{p}^{-1 / 2}=p^{1 / 2}, \gamma(f)=\gamma(-\eta)=\frac{1}{ \pm \sigma_{p}}$ (see Lemma 2) and $\rho\left(\pi_{\eta}, s\right)= \pm \sigma_{p} p^{s-\frac{1}{2}}$ (see Lemma 4). Then

$$
\begin{aligned}
\rho\left(\pi_{1}, s\right) \rho\left(\pi_{-D}, s\right)|D|_{p}^{-1 / 2} \gamma(f)|\eta|_{p}^{s} & =\frac{1-p^{s-1}}{1-p^{-s}}\left( \pm \sigma_{p}\right) p^{s-\frac{1}{2}} p^{1 / 2}\left(\frac{1}{ \pm \sigma_{p}}\right) p^{-s} \\
& =\frac{1-p^{s-1}}{1-p^{-s}} .
\end{aligned}
$$

Proposition 2. Take $f(x)=x_{1}^{2}-a x_{2}^{2}-p x_{3}^{2}+a p x_{4}^{2}$, with $a \in \mathbb{Z}$ a quadratic non-residue module $p$. Then

$$
\int_{\mathbb{Q}_{p}^{4} \backslash\{0\}}|f(x)|^{s-2} \widehat{\varphi}(x) d^{4} x=\frac{1-p^{s-2}}{\left(1-p^{-s}\right)} \int_{\mathbb{Q}_{p}^{4} \backslash\{0\}}\left|a p x_{1}^{2}-p x_{2}^{2}-a x_{3}^{2}+x_{4}^{2}\right|_{p}^{-s} \varphi(x) d^{4} x .
$$


Proof. In this case $n=4, D=p^{2} a^{2}, D^{*}=D$ and $\gamma(f)=(a, p)_{p}=-1$, see Lemma 3 (ii) and (2.4), the functional equation takes the form

$$
\begin{aligned}
& \int_{\mathbb{Q}_{p}^{4} \backslash\{0\}}|f(x)|_{p}^{s-2} \widehat{\varphi}(x) d^{4} x \\
= & -\rho\left(\pi_{1}, s-1\right) \rho\left(\pi_{1}, s\right) p \int_{\mathbb{Q}_{p}^{4} \backslash\{0\}}\left|x_{1}^{2}-a^{-1} x_{2}^{2}-p^{-1} x_{3}^{2}+(a p)^{-1} x_{4}^{2}\right|_{p}^{-s} \varphi(x) d^{4} x \\
= & -|a p|_{p}^{s} p \frac{1-p^{s-2}}{1-p^{1-s}} \frac{1-p^{s-1}}{1-p^{-s}} \int_{\mathbb{Q}_{p}^{4} \backslash\{0\}}\left|a p x_{1}^{2}-p x_{2}^{2}-a x_{3}^{2}+x_{4}^{2}\right|_{p}^{-s} \varphi(x) d^{4} x \\
= & \frac{1-p^{s-2}}{\left(1-p^{-s}\right)} \int_{\mathbb{Q}_{p}^{4} \backslash\{0\}}\left|a p x_{1}^{2}-p x_{2}^{2}-a x_{3}^{2}+x_{4}^{2}\right|_{p}^{-s} \varphi(x) d^{4} x .
\end{aligned}
$$

\section{Riesz Kernels and Lizorkin Spaces of Second Kind}

In this section we introduce a new type of Riesz kernels depending on a complex parameter and a certain quadratic form. The main result of this section establishes that these kernels considered as distributions on a Lizorkin spaces of second kind form an Abelian group under convolution.

3.1. Riesz Kernels. In this section $f(x):=x_{1}^{2}-a x_{2}^{2}-p x_{3}^{2}+a p x_{4}^{2}$ with $a \in \mathbb{Z}$ a quadratic non-residue module $p$. Note that $a \in \mathbb{Z}_{p}^{\times}$and that $f(x)$ is an elliptic quadratic form, i.e. $f(x)=0 \Leftrightarrow x=0$. We call the function

$$
K_{\alpha}(x):=\frac{1-p^{-\alpha}}{1-p^{\alpha-2}}|f(x)|_{p}^{\alpha-2}, \quad \operatorname{Re}(\alpha)>0, \alpha \neq 2+\frac{2 \pi \sqrt{-1}}{\ln p} \mathbb{Z},
$$

the Riesz kernel attached to $f(x)$.

Lemma 5. Set $\varphi$ to be the characteristic function of the ball $\widetilde{x}_{0}+\left(p^{m} \mathbb{Z}_{p}\right)^{4}$. Then

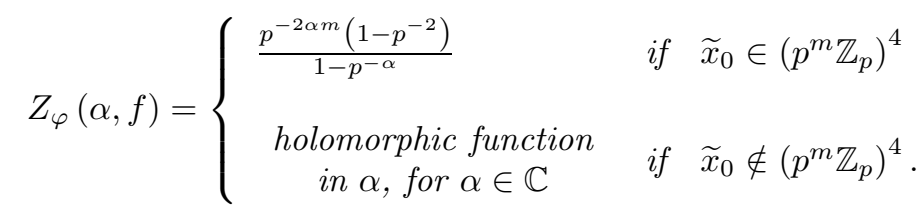

Proof. We consider first the case $\widetilde{x}_{0} \in\left(p^{m} \mathbb{Z}_{p}\right)^{4}$. Set

$$
Z(\alpha):=\int_{\mathbb{Z}_{p}^{4} \backslash\{0\}}|f(x)|_{p}^{\alpha-2} d^{4} x \text { for } \operatorname{Re}(\alpha)>2 .
$$

By a change of variables $Z_{\varphi}(\alpha, f)=p^{-2 \alpha m} Z(\alpha)$. The result follows from the following formula:

$$
Z(\alpha)=\frac{1-p^{-2}}{1-p^{-\alpha}} \text { for } \operatorname{Re}(\alpha)>2
$$


Set $\mathbb{Z}_{p}^{4}=\left(p \mathbb{Z}_{p}\right)^{4} \bigsqcup U$ with $U=\left\{x \in \mathbb{Z}_{p}^{4}:\|x\|_{p}=1\right\}$. Then

$$
\begin{aligned}
Z(\alpha) & =\int_{\left(p \mathbb{Z}_{p}\right)^{4}}|f(x)|_{p}^{\alpha-2} d^{4} x+\int_{U}|f(x)|_{p}^{\alpha-2} d^{4} x \\
& =p^{-2 \alpha} Z(\alpha)+\int_{U}|f(x)|_{p}^{\alpha-2} d^{4} x,
\end{aligned}
$$

i.e.

$$
Z(\alpha)=\frac{1}{1-p^{-2 \alpha}} \int_{U}|f(x)|_{p}^{\alpha-2} d^{4} x .
$$

In order to show (3.1), it is sufficient to prove the following formula:

$$
\int_{U}|f(x)|_{p}^{\alpha-2} d^{4} x=\left(1-p^{-2}\right)\left(1+p^{-\alpha}\right) \text { for } \alpha \in \mathbb{C} .
$$

This formula can be established as follows. For $i=\left(i_{1}, i_{2}, i_{3}, i_{4}\right) \in\{0,1\}^{4} \backslash$ $\{(1,1,1,1)\}$ we define

$$
\begin{aligned}
& U^{(i)}=U_{1}^{(i)} \times U_{2}^{(i)} \times U_{3}^{(i)} \times U_{4}^{(i)}, \\
& U_{j}^{(i)}:=\left\{\begin{array}{lll}
p \mathbb{Z}_{p} & \text { if } & i_{j}=1 \\
& & \\
\mathbb{Z}_{p}^{\times} & \text {if } & i_{j}=0 .
\end{array}\right.
\end{aligned}
$$

Then $U=\bigsqcup_{i} U^{(i)}$ and

$$
\int_{U}|f(x)|_{p}^{\alpha-2} d^{4} x=\sum_{i} \int_{U^{(i)}}|f(x)|_{p}^{\alpha-2} d^{4} x:=\sum_{i} Z_{i}(\alpha) .
$$

By a direct calculation one finds:

\begin{tabular}{|c|l|}
\hline Index $i$ & $Z_{i}(\alpha)$ \\
\hline$(1,1,1,0)$ & $p^{-\alpha-1}\left(1-p^{-1}\right)$ \\
\hline$(1,1,0,1)$ & $p^{-\alpha-1}\left(1-p^{-1}\right)$ \\
\hline$(1,1,0,0)$ & $p^{-\alpha}\left(1-p^{-1}\right)^{2}$ \\
\hline$(1,0,1,1), \quad(0,1,1,1)$ & $\left(1-p^{-1}\right) p^{-3}$ \\
\hline $\begin{array}{c}(1,0,1,0), \quad(1,0,0,1), \quad(0,1,1,0) \\
(0,1,0,1), \quad(0,0,1,1)\end{array}$ & $\left(1-p^{-1}\right)^{2} p^{-2}$ \\
\hline $\begin{array}{c}(1,0,0,0), \quad(0,1,0,0), \quad(0,0,1,0) \\
(0,0,0,1)\end{array}$ & $\left(1-p^{-1}\right)^{3} p^{-1}$ \\
\hline$(0,0,0,0)$ & $\left(1-p^{-1}\right)^{4}$ \\
\hline
\end{tabular}

In the case $\widetilde{x}_{0} \notin\left(p^{m} \mathbb{Z}_{p}\right)^{4}, f$ does not vanish on the ball $\widetilde{x}_{0}+\left(p^{m} \mathbb{Z}_{p}\right)^{4}$ which implies that $Z_{\varphi}(\alpha)$ is a holomorphic function on the whole complex plane. 
Lemma 6. $K_{\alpha}(x)$ possesses, as a distribution on $S\left(\mathbb{Q}_{p}^{4}\right)$, a meromorphic continuation to all $\alpha \neq 2+\frac{2 \pi \sqrt{-1}}{\ln p} \mathbb{Z}$ given by

$$
\begin{aligned}
& \left\langle K_{\alpha}, \varphi\right\rangle=\varphi(0) \frac{1-p^{-2}}{1-p^{\alpha-2}}+ \\
& \frac{1-p^{-\alpha}}{1-p^{\alpha-2}}\left[\int_{|| x \|_{p}>1} \varphi(x)|f(x)|_{p}^{\alpha-2} d^{4} x+\int_{\|x\|_{p} \leq 1}(\varphi(x)-\varphi(0))|f(x)|_{p}^{\alpha-2} d^{4} x\right] .
\end{aligned}
$$

Proof. The result follows from Lemma 5 by

$$
\begin{aligned}
& \left\langle K_{\alpha}, \varphi\right\rangle=\frac{1-p^{-\alpha}}{1-p^{\alpha-2}} \int_{\mathbb{Q}_{p}^{4} \backslash\{0\}} \varphi(x)|f(x)|_{p}^{\alpha-2} d^{4} x= \\
& \frac{1-p^{-\alpha}}{1-p^{\alpha-2}}\left[\int_{|| x \|_{p}>1} \varphi(x)|f(x)|_{p}^{\alpha-2} d^{4} x+\int_{\|x\|_{p} \leq 1}(\varphi(x)-\varphi(0))|f(x)|_{p}^{\alpha-2} d^{4} x\right] \\
& +\varphi(0) \frac{1-p^{-2}}{1-p^{\alpha-2}} .
\end{aligned}
$$

From Lemma 6 follows that the distribution $K_{\alpha}$ has simple poles at the points $\alpha=2+\alpha_{k}$ with $\alpha_{k}:=\frac{2 k \pi \sqrt{-1}}{\ln p}, \quad k \in \mathbb{Z}$, and

$$
\lim _{\alpha \rightarrow \alpha_{k}} K_{\alpha}:=K_{\alpha_{k}}=\delta
$$

where $\delta$ denotes the Dirac distribution.

LeMma 7.

$$
\int_{\|x\|_{p}>1} \frac{1}{|f(x)|_{p}^{\alpha+2}} d x=\frac{p^{-2 \alpha}\left(1-p^{-2}\right)\left(1+p^{\alpha}\right)}{1-p^{-2 \alpha}}, \quad \operatorname{Re}(\alpha)>0 .
$$

Proof. Set $U=\left(\mathbb{Z}_{p}\right)^{4} \backslash\left(p \mathbb{Z}_{p}\right)^{4}$ as before. Then

$$
\begin{aligned}
& \int_{\|x\|_{p}>1} \frac{1}{|f(x)|_{p}^{\alpha+2}} d^{4} x=\sum_{m=1}^{\infty} \int_{p^{-m} U} \frac{1}{|f(x)|^{\alpha+2}} d^{4} x \\
&=\sum_{m=1}^{\infty} p^{-2 m \alpha} \int_{U} \frac{1}{|f(x)|_{p}^{\alpha+2}} d^{4} x=\frac{p^{-2 \alpha}}{1-p^{-2 \alpha}} \int_{U} \frac{1}{|f(x)|_{p}^{\alpha+2}} d^{4} x \\
& \quad=\frac{p^{-2 \alpha}\left(1-p^{-2}\right)\left(1+p^{\alpha}\right)}{1-p^{-2 \alpha}}
\end{aligned}
$$

where we used (3.2).

Proposition 3. For $\operatorname{Re}(\alpha)>0$ and $\varphi \in \mathbf{S}\left(\mathbb{Q}_{p}^{4}\right)$, the following formulas hold:

(i) $\left\langle K_{\alpha}, \varphi\right\rangle=\frac{1-p^{-\alpha}}{1-p^{\alpha-2}} \int_{\mathbb{Q}_{p}^{4} \backslash\{0\}}|f(x)|_{p}^{\alpha-2} \varphi(x) d^{4} x, \quad \alpha \neq 2+\alpha_{k}$;

(ii) $\left\langle K_{-\alpha}, \varphi\right\rangle=\frac{1-p^{\alpha}}{1-p^{-\alpha-2}} \int_{\mathbb{Q}_{p}^{4}} \frac{\varphi(x)-\varphi(0)}{|f(x)|_{p}^{\alpha+2}} d^{4} x$;

(iii) $\left(K_{\alpha} * \varphi\right)(x)=\frac{1-p^{-\alpha}}{1-p^{\alpha-2}} \int_{\mathbb{Q}_{p}^{4} \backslash\{0\}}|f(y)|_{p}^{\alpha-2} \varphi(x+y) d^{4} y, \quad \alpha \neq 2+\alpha_{k} ;$ 
(iv) $\left(K_{-\alpha} * \varphi\right)(x)=\frac{1-p^{\alpha}}{1-p^{-\alpha-2}} \int_{\mathbb{Q}_{p}^{4}} \frac{\varphi(x+y)-\varphi(x)}{|f(y)|_{p}^{\alpha+2}} d^{4} y$.

Proof. (i) Since every test function can be written a finite sums of characteristic functions of balls, Lemma 5 implies that

$$
\frac{1-p^{-\alpha}}{1-p^{\alpha-2}} \int_{\mathbb{Q}_{p}^{4} \backslash\{0\}}|f(x)|_{p}^{\alpha-2} \varphi(x) d^{4} x
$$

is well-defined for $\operatorname{Re}(\alpha)>0$ and $\alpha \neq 2+\alpha_{k}$. The announced formula follows by a calculation similar to the one done in the proof of Lemma 6 .

(ii) We first note that the integral

$$
\frac{1-p^{\alpha}}{1-p^{-\alpha-2}} \int_{\mathbb{Q}_{p}^{4}} \frac{\varphi(x)-\varphi(0)}{|f(x)|_{p}^{\alpha+2}} d^{4} x
$$

converges on $\operatorname{Re}(\alpha)>0$. Indeed, since $f(x)$ is an elliptic quadratic form we have

$$
B\|x\|_{p}^{2} \leq|f(x)|_{p} \leq A\|x\|_{p}^{2} \text { for any } x \in \mathbb{Q}_{p}^{n}
$$

where $A, B$ are positive constants, cf. [25. Lemma 1], then

$$
\int_{\mathbb{Q}_{p}^{4}} \frac{|\varphi(x)-\varphi(0)|}{|f(x)|_{p}^{\operatorname{Re}(\alpha)+2}} d^{4} x \leq \frac{2\|\varphi\|_{L^{\infty}}}{B^{\operatorname{Re}(\alpha)+2}} \int_{\|x\|_{p}>p^{m}} \frac{1}{\|x\|_{p}^{2 \operatorname{Re}(\alpha)+4}} d^{4} x<\infty,
$$

where $m$ is the exponent of local constancy of $\varphi$.

Now

$$
\begin{aligned}
& \frac{1-p^{\alpha}}{1-p^{-\alpha-2}} \int_{\mathbb{Q}_{p}^{4}} \frac{\varphi(x)-\varphi(0)}{|f(x)|_{p}^{\alpha+2}} d^{4} x= \\
& \frac{1-p^{\alpha}}{1-p^{-\alpha-2}}\left\{\int_{\|x\|_{p} \leq 1} \frac{\varphi(x)-\varphi(0)}{|f(x)|_{p}^{2+\alpha}} d^{4} x+\int_{\|\left. x\right|_{p}>1} \frac{\varphi(x)}{|f(x)|_{p}^{2+\alpha}} d^{4} x\right\}-\varphi(0) \frac{1-p^{\alpha}}{1-p^{-\alpha-2}} \\
& \quad \times \int_{\|x\|_{p}>1} \frac{1}{|f(x)|_{p}^{2+\alpha}} d^{4} x \\
& =\frac{1-p^{\alpha}}{1-p^{-\alpha-2}}\left\{\int_{\mid x \|_{p} \leq 1} \frac{\varphi(x)-\varphi(0)}{\left.|f(x)|_{p}^{2+\alpha} d^{4} x+\int_{\|\left. x\right|_{p}>1} \frac{\varphi(x)}{|f(x)|_{p}^{2+\alpha}} d^{4} x\right\}}\right. \\
& -\varphi(0) \frac{1-p^{\alpha}}{1-p^{-\alpha-2}} \frac{p^{-2 \alpha}\left(1-p^{-2}\right)\left(1+p^{\alpha}\right)}{1-p^{-2 \alpha}}=\left\langle K_{-\alpha}, \varphi\right\rangle,
\end{aligned}
$$

where we used Lemmas 7 and 6 .

(iii)-(iv) We recall that if $\varphi \in \mathbf{S}\left(\mathbb{Q}_{p}^{4}\right)$, then $\left(K_{\alpha} * \varphi\right)(x)=\left\langle K_{\alpha}(y), \varphi(x-y)\right\rangle$, and since $K_{\alpha}(-y)=K_{\alpha}(y)$, we have $\left(K_{\alpha} * \varphi\right)(x)=\left\langle K_{\alpha}(y), \varphi(x+y)\right\rangle$. Therefore (iii) follows from (i) and (iv) follows from (ii).

3.2. Lizorkin spaces of second kind. Consider the spaces

$$
\boldsymbol{\Psi}:=\boldsymbol{\Psi}\left(\mathbb{Q}_{p}^{n}\right)=\left\{\psi \in \boldsymbol{S}\left(\mathbb{Q}_{p}^{n}\right) \mid \psi(0)=0\right\}
$$

and

$$
\boldsymbol{\Phi}:=\boldsymbol{\Phi}\left(\mathbb{Q}_{p}^{n}\right)=\left\{\phi \mid \phi=\mathcal{F}[\psi], \quad \psi \in \boldsymbol{\Psi}\left(\mathbb{Q}_{p}^{n}\right)\right\}
$$


The space $\mathbf{\Phi}$ is called the p-adic Lizorkin space of test functions of second kind. We equip $\boldsymbol{\Psi}$ and $\boldsymbol{\Phi}$ with the topology inherited from $\boldsymbol{S}\left(\mathbb{Q}_{p}^{n}\right)$. Note that $\mathcal{F}: \boldsymbol{\Psi} \rightarrow \boldsymbol{\Phi}$ is an isomorphism of linear spaces and $\mathcal{F}(\mathcal{F}[\Psi])=\boldsymbol{\Psi}$.

Let $\boldsymbol{\Phi}^{\prime}=\boldsymbol{\Phi}^{\prime}\left(\mathbb{Q}_{p}^{n}\right)$ denote the topological dual of the space $\mathbf{\Phi}\left(\mathbb{Q}_{p}^{n}\right)$. This is space of the p-adic Lizorkin space of distributions of the second kind. by

We define the Fourier transform of distributions $J \in \Phi^{\prime}\left(\mathbb{Q}_{p}^{n}\right)$ and $G \in \Psi^{\prime}\left(\mathbb{Q}_{p}^{n}\right)$

$$
\begin{aligned}
& \langle\mathcal{F}[J], \psi\rangle=\langle J, \mathcal{F}[\psi]\rangle, \quad \text { for any } \psi \in \boldsymbol{\Psi}\left(\mathbb{Q}_{p}^{n}\right), \\
& \langle\mathcal{F}[G], \phi\rangle=\langle G, \mathcal{F}[\phi]\rangle, \quad \text { for any } \phi \in \mathbf{\Phi}\left(\mathbb{Q}_{p}^{n}\right) .
\end{aligned}
$$

It is clear that a $\mathcal{F}\left[\Psi^{\prime}\left(\mathbb{Q}_{p}^{n}\right)\right]=\boldsymbol{\Phi}^{\prime}\left(\mathbb{Q}_{p}^{n}\right)$ and $\mathcal{F}\left[\Phi^{\prime}\left(\mathbb{Q}_{p}^{n}\right)\right]=\boldsymbol{\Psi}^{\prime}\left(\mathbb{Q}_{p}^{n}\right)$. For further details about $p$-adic Lizorkin spaces the reader may consult [1].

3.3. The Riesz kernels form an Abelian group. The goal of this section is to prove the following result:

Theorem 2. For $\alpha, \beta \in \mathbb{C}, K_{\alpha} * K_{\beta}=K_{\alpha+\beta}$ in $\boldsymbol{\Phi}^{\prime}\left(\mathbb{Q}_{p}^{4}\right)$.

Before giving the proof we need to establish several auxiliary results.

Definition 1. Set $f^{\circ}(x):=a p x_{1}^{2}-p x_{2}^{2}-a x_{3}^{2}+x_{4}^{2}$. The Riesz kernel attached to $f^{\circ}(x)$ is the distribution

$$
K_{-\alpha}^{\circ}(x):=\left|f^{\circ}(x)\right|_{p}^{-\alpha} \text { in } \Psi^{\prime}\left(\mathbb{Q}_{p}^{4}\right), \text { for } \alpha \in \mathbb{C} .
$$

Proposition 4. Considering $K_{\alpha} \in \boldsymbol{\Phi}^{\prime}\left(\mathbb{Q}_{p}^{4}\right)$ and $K_{-\alpha}^{\circ} \in \boldsymbol{\Psi}^{\prime}\left(\mathbb{Q}_{p}^{4}\right)$, we have

$$
\mathcal{F}\left[K_{\alpha}\right]=K_{-\alpha}^{\circ} \text { for } \alpha \neq 2+\alpha_{k} \text { and } \alpha \neq \alpha_{k}, k \in \mathbb{Z} .
$$

Proof. The formula follows from Proposition 2

LEMMA 8.

$$
\lim _{\alpha \rightarrow 2+\alpha_{k}}\left\langle K_{\alpha}, \varphi\right\rangle=-\frac{1-p^{-2}}{\ln p}\left\langle\ln |f(x)|_{p}, \varphi(x)\right\rangle \text { for } \varphi \in \mathbf{\Phi}\left(\mathbb{Q}_{p}^{4}\right) .
$$

REMARK 1. We understand the right-hand side in (3.5) as the distribution induced by the locally integrable function $\ln |f(x)|_{p}: \mathbb{Q}_{p}^{4} \backslash\{0\} \rightarrow \mathbb{R}$.

Proof. Since

$$
\begin{aligned}
\lim _{\alpha \rightarrow 2+\alpha_{k}}\left\langle K_{\alpha}, \varphi\right\rangle=\lim _{\alpha \rightarrow 2+\alpha_{k}} \frac{1-p^{-\alpha}}{1-p^{\alpha-2}} \int_{\mathbb{Q}_{p}^{4} \backslash\{0\}}|f(x)|_{p}^{\alpha-2} \varphi(x) d^{4} x \\
=\lim _{\beta \rightarrow 2}\left(1-p^{-\beta}\right) \int_{\mathbb{Q}_{p}^{4} \backslash\{0\}}\left[\frac{\left|x_{1}^{2}-a x_{2}^{2}-p x_{3}^{2}+a p x_{4}^{2}\right|_{p}^{\beta-2}-1}{1-p^{\beta-2}}\right] \varphi(x) d^{4} x
\end{aligned}
$$

by taking $\beta=\alpha-\alpha_{k}$ and by using the fact that $\int \varphi(x) d^{4} x=0$. Now by passing to the limit under the integral sign we have

$$
\lim _{\alpha \rightarrow 2+\alpha_{k}}\left\langle K_{\alpha}, \varphi\right\rangle=-\left(1-p^{-2}\right) \int_{\mathbb{Q}_{p}^{4} \backslash\{0\}} \frac{\ln |f(x)|_{p}}{\ln p} \varphi(x) d^{4} x .
$$


The passage to the limit under the integral sign is justified by the Lebesgue Dominated Convergence Theorem and the inequality

$$
\left|\frac{e^{(\beta-2) \ln |f(x)|_{p}}-1}{1-e^{(\beta-2) \ln p}}\right| \leq C\left|\frac{\ln |f(x)|_{p}}{\ln p}\right| \text { for } x \in \operatorname{supp} \varphi \subset \mathbb{Q}_{p}^{4} \backslash\{0\} \text { and }|\beta-2| \leq 1,
$$

where $C=C(p, \operatorname{supp} \varphi)$ is a positive constant.

Definition 2. We define

$$
K_{2+\alpha_{k}}(x)=-\frac{1-p^{-2}}{\ln p} \ln |f(x)|_{p} \in \boldsymbol{\Phi}^{\prime}\left(\mathbb{Q}_{p}^{4}\right) .
$$

Lemma 9.

$$
\left\langle\mathcal{F}\left[K_{2+\alpha_{k}}\right], \varphi\right\rangle=\left\langle K_{-2}^{\circ}, \varphi\right\rangle, \quad \text { for } \varphi \in \Psi\left(\mathbb{Q}_{p}^{4}\right) .
$$

Proof. By using the fact that $K_{2+\alpha_{k}}=K_{2}$ and by Proposition 4 we get

$$
\left\langle\mathcal{F}\left[K_{2+\alpha_{k}}\right], \varphi\right\rangle=\lim _{\alpha \rightarrow 2+\alpha_{k}}\left\langle K_{\alpha}, \mathcal{F}[\varphi]\right\rangle=\lim _{\alpha \rightarrow 2}\left\langle K_{\alpha}, \mathcal{F}[\varphi]\right\rangle=\lim _{\alpha \rightarrow 2}\left\langle K_{-\alpha}^{\circ}, \varphi\right\rangle .
$$

By using $\varphi(0)=0$, we have

$$
\lim _{\alpha \rightarrow 2}\left\langle K_{\alpha}^{\circ}, \varphi\right\rangle=\lim _{\alpha \rightarrow 2} \int_{\|x\|_{p}>p^{m}}\left|f^{\circ}(x)\right|_{p}^{-\alpha} \varphi(x) d^{4} x,
$$

where $m \in \mathbb{Z}$ is the exponent of local constancy of $\varphi$. We now use the fact that $f^{\circ}(x)$ is an elliptic quadratic form to get

$$
B\|x\|_{p}^{2} \leq\left|f^{\circ}(x)\right|_{p} \leq A\|x\|_{p}^{2} \text { for any } x \in \mathbb{Q}_{p}^{n},
$$

where $A, B$ are positive constants, cf. [25, Lemma 1]. Without loss of generality we may assume that $B \leq 1$ and that $m>0$. Then

$$
\left|f^{\circ}(x)\right|_{p}^{-\operatorname{Re}(\alpha)}|\varphi(x)| \leq \frac{|\varphi(x)|}{B^{\operatorname{Re}(\alpha)}\|x\|_{p}^{2 \operatorname{Re}(\alpha)}} \leq \frac{|\varphi(x)|}{B^{2+\epsilon} p^{2 m(2-\epsilon)}}
$$

which is an integrable function on $\left(\mathbb{Q}_{p}^{4} \backslash B_{m}(0)\right) \cap \operatorname{supp} \varphi$ and $\alpha \in(2-\epsilon, 2+\epsilon)$, where $\epsilon$ is a small fixed positive constant. Therefore, by applying the Lebesgue Dominated Convergence Theorem,

$$
\lim _{\alpha \rightarrow 2}\left\langle K_{\alpha}^{\circ}, \varphi\right\rangle=\int_{\mathbb{Q}_{p}^{4}}\left|f^{\circ}(x)\right|_{p}^{-2} \varphi(x) d^{4} x .
$$

Proposition 5. Considering $K_{\alpha} \in \boldsymbol{\Phi}^{\prime}\left(\mathbb{Q}_{p}^{4}\right)$ and $K_{-\alpha}^{\circ} \in \boldsymbol{\Psi}^{\prime}\left(\mathbb{Q}_{p}^{4}\right)$,

$$
\mathcal{F}\left[K_{\alpha}\right]=K_{-\alpha}^{\circ} \text { for } \alpha \in \mathbb{C} .
$$

Proof. The result follows from Proposition 4 and Lemma 9 ,

Lemma 10. For any $\alpha, \beta \in \mathbb{C}, K_{-\alpha}^{\circ} \cdot K_{-\beta}^{\circ}=K_{-(\alpha+\beta)}^{\circ}$ in $\in \mathbf{\Psi}^{\prime}\left(\mathbb{Q}_{p}^{4}\right)$. 
Proof. It follows from Lemma 1 Indeed, the functions $K_{-\alpha}^{\circ}$ and $K_{-\beta}^{\circ}$ belong to $L_{l o c}^{1}$, and

$$
\lim _{\xi \rightarrow 0} \int_{\mathbb{Q}_{p}^{4}} K_{-\alpha}^{\circ}(x) \varphi(x) K_{-\beta}^{\circ}(x-\xi) d^{4} x=\int_{\mathbb{Q}_{p}^{4}} K_{-\alpha}^{\circ}(x) \varphi(x) K_{-\beta}^{\circ}(x) d^{4} x .
$$

This last statement follows from the Lebesgue Dominated Convergence Theorem and (3.6) by the inequality

$$
\left|K_{-\alpha}^{\circ}(x) \varphi(x) K_{-\beta}^{\circ}(x-\xi)\right| \leq C(\varphi, \alpha, \beta)\|x\|_{p}^{-2 \operatorname{Re}(\alpha)-2 \operatorname{Re}(\beta)}
$$

for $x \in \operatorname{supp} \varphi$ and $\|\xi\|_{p} \leq p^{m(\varphi)}$, where $C(\varphi, \alpha, \beta)$ is a positive constant and $m(\varphi)$ is the largest integer satisfying $\left.\varphi\right|_{B_{m(\varphi)}(0)} \equiv 0$.

3.3.1. Proof of Theorem 囵. By Lemma 10, for any $\alpha, \beta \in \mathbb{C}$, we have

$$
\mathcal{F}\left[K_{-\alpha}^{\circ} \cdot K_{-\beta}^{\circ}\right]=\mathcal{F}\left[K_{-\alpha}^{\circ}\right] * \mathcal{F}\left[K_{-\beta}^{\circ}\right]=\mathcal{F}\left[K_{-(\alpha+\beta)}^{\circ}\right] \text { in } \boldsymbol{\Phi}^{\prime}\left(\mathbb{Q}_{p}^{4}\right) .
$$

By Proposition $\left[K_{\alpha}=\mathcal{F}\left[K_{-\alpha}^{\circ}\right]\right.$ for $\alpha \in \mathbb{C}$ since $\mathcal{F}\left[\mathcal{F}\left[K_{\alpha}\right]\right]=K_{\alpha}$, therefore for any $\alpha, \beta \in \mathbb{C}, K_{\alpha} * K_{\beta}=K_{\alpha+\beta}$.

REMARK 2. The proof given Theorem 2 can be extended to cover the elliptic quadratic forms of dimension 2, see Proposition 1 .

\section{Pseudodifferential Operators and Fundamental Solutions}

We take $f(\xi)=\xi_{1}^{2}-a \xi_{2}^{2}-p \xi_{3}^{2}+a p \xi_{4}^{2}, f^{*}(\xi)=\frac{a p \xi_{1}^{2}-p \xi_{2}^{2}-a \xi_{3}^{2}+\xi_{4}^{2}}{a p}$, with $a \in \mathbb{Z}$ a quadratic non-residue module $p$, as in Section 3 Given $\alpha>0$, we define the pseudodifferential operator with symbol $\left|f^{\circ}(\xi)\right|_{p}^{\alpha}$ by

$$
\begin{array}{ll}
\mathbf{S}\left(\mathbb{Q}_{p}^{4}\right) & \rightarrow C\left(\mathbb{Q}_{p}^{4}\right) \cap L^{2}\left(\mathbb{Q}_{p}^{4}\right) \\
\varphi & \rightarrow(\boldsymbol{f}(\partial, \alpha) \varphi)(x):=\mathcal{F}_{\xi \rightarrow x}^{-1}\left(\left|f^{\circ}(\xi)\right|_{p}^{\alpha} \mathcal{F}_{x \rightarrow \xi} \varphi\right) .
\end{array}
$$

This operator is well-defined since $\left|f^{\circ}(\xi)\right|_{p}^{\alpha} \mathcal{F}_{x \rightarrow \xi} \varphi \in L^{1}\left(\mathbb{Q}_{p}^{4}\right) \cap L^{2}\left(\mathbb{Q}_{p}^{4}\right)$. Note that $\left|p f^{*}(\xi)\right|_{p}^{\alpha}=\left|f^{\circ}(\xi)\right|_{p}^{\alpha}$. Since $\mathcal{F}^{-1}\left(\left|f^{\circ}\right|_{p}^{\alpha}\right)=K_{\alpha}$ (cf. Proposition 5 ), by applying Proposition 3 (iv), we get

$$
\boldsymbol{f}(\partial, \alpha) \varphi=K_{-\alpha} * \varphi=\frac{1-p^{\alpha}}{1-p^{-\alpha-2}} \int_{\mathbb{Q}_{p}^{4}} \frac{\varphi(x-y)-\varphi(x)}{|f(y)|_{p}^{\alpha+2}} d^{4} y
$$

for $\varphi \in \mathbf{S}\left(\mathbb{Q}_{p}^{4}\right)$. isfying

Set $\mathcal{E}_{f, \alpha}\left(\mathbb{Q}_{p}^{4}\right)$ to be the class consisting of locally constant functions $\varphi(x)$ sat-

$$
\int_{\|x\|_{p} \geq p^{m}} \frac{|\varphi(x)|}{|f(x)|_{p}^{\alpha+2}} d^{4} x<\infty \text { for some } m \in \mathbb{Z} .
$$

The operator $\boldsymbol{f}(\partial, \alpha)$ can be extended to $\mathcal{E}_{f, \alpha}\left(\mathbb{Q}_{p}^{4}\right)$.

Lemma 11. If $\varphi \in \mathcal{E}_{f, \alpha}\left(\mathbb{Q}_{p}^{4}\right)$, then the integral on the right- hand side of (4.1) converges. 
Proof. Since $\varphi$ is locally constant there exists $l=l(x) \in \mathbb{Z}$ such that $\varphi(x-$ $y)-\varphi(x)=0$ for $\|y\|_{p} \leq p^{l}$, thus, it is sufficient to show the convergence of the following integrals:

$$
\int_{\|y\|_{p}>p^{l}} \frac{1}{|f(y)|_{p}^{\alpha+2}} d^{4} y, \int_{\|y\|_{p}>p^{l}} \frac{|\varphi(x-y)|}{|f(y)|_{p}^{\alpha+2}} d^{4} y=\int_{\|x-z\|_{p}>p^{l}} \frac{|\varphi(z)|}{|f(x-z)|_{p}^{\alpha+2}} d^{4} z .
$$

The convergence of the first integral follows from (3.4). To establish the convergence of the second integral, it is sufficient to show the convergence of the integral

$$
\int_{\|x-z\|_{p}>p^{l}} \frac{|\varphi(z)|}{\|x-z\|_{p}^{2 \alpha+4}} d^{4} z
$$

cf. (3.4). The convergence of this last integral is established by considering the cases: (i) $\|x\|_{p}<\|z\|_{p}$, (ii) $\|x\|_{p}>\|z\|_{p}$, (iii) $\|x\|_{p}=\|z\|_{p}$. The verification of cases (i)-(ii) is left to the reader. In the case (iii), we change variables as $x=p^{M} \widetilde{x}$, $z=p^{M} \widetilde{z}$ with $\|\widetilde{x}\|_{p}=\|\widetilde{z}\|_{p}=1$ in (4.2), then

$$
\begin{aligned}
\int_{\|x-z\|_{p}>p^{l}} \frac{|\varphi(z)|}{\|x-z\|_{p}^{2 \alpha+4}} d^{4} z & =p^{2 M \alpha} \int_{\substack{\|\widetilde{x}-\widetilde{z}\|_{p}>p^{l+M} \\
\|\widetilde{z}\|=1}} \frac{\left|\varphi\left(p^{M} \widetilde{z}\right)\right|}{\|\widetilde{x}-\widetilde{z}\|_{p}^{2 \alpha+4}} d^{4} \widetilde{z} \\
& \leq p^{2 M \alpha-(2 \alpha+4)(l+M)} \int_{\|\widetilde{z}\|=1}\left|\varphi\left(p^{M} \widetilde{z}\right)\right| d^{4} \widetilde{z}<\infty
\end{aligned}
$$

The space of test functions $\mathbf{S}\left(\mathbb{Q}_{p}^{4}\right)$ is not invariant under the action of $\boldsymbol{f}(\partial, \alpha)$. But if we replace $\mathbf{S}\left(\mathbb{Q}_{p}^{4}\right)$ by $\boldsymbol{\Phi}\left(\mathbb{Q}_{p}^{4}\right)$ then $\boldsymbol{f}(\partial, \alpha) \boldsymbol{\Phi}\left(\mathbb{Q}_{p}^{4}\right)=\boldsymbol{\Phi}\left(\mathbb{Q}_{p}^{4}\right)$. The verification of this fact involves the same ideas used in the verification of the corresponding assertion for the Taibleson operator, see e.g. [1, Lemma 9.2.5]. On the other hand, the mapping

$$
\begin{array}{ccc}
\boldsymbol{\Phi}^{\prime}\left(\mathbb{Q}_{p}^{4}\right) & \rightarrow & \boldsymbol{\Phi}^{\prime}\left(\mathbb{Q}_{p}^{4}\right) \\
J & \rightarrow & \boldsymbol{f}(\partial, \alpha) J:=\mathcal{F}^{-1}\left[\left|f^{\circ}\right|_{p}^{\alpha} \mathcal{F}[J]\right]
\end{array}
$$

is a homeomorphism. This is a consequence of the fact that the map

$$
\begin{array}{ll}
\boldsymbol{\Psi}\left(\mathbb{Q}_{p}^{4}\right) & \rightarrow \boldsymbol{\Psi}\left(\mathbb{Q}_{p}^{4}\right) \\
\varphi & \rightarrow\left|p f^{*}\right|_{p}^{\alpha} \varphi
\end{array}
$$

is a homeomorphism.

LEMMA 12. The following formulas hold:

(i) $\langle\boldsymbol{f}(\partial, \alpha) J, \varphi\rangle=\langle J, \boldsymbol{f}(\partial, \alpha) \varphi\rangle$ for any $J \in \boldsymbol{\Phi}^{\prime}\left(\mathbb{Q}_{p}^{4}\right)$ and $\varphi \in \boldsymbol{\Phi}\left(\mathbb{Q}_{p}^{4}\right)$;

(ii) $\boldsymbol{f}(\partial, \alpha) J=K_{-\alpha} * J$ for any $J \in \mathbf{\Phi}^{\prime}\left(\mathbb{Q}_{p}^{4}\right)$. 
Proof. (i) The formula follows from the following calculation:

$$
\begin{aligned}
\langle\boldsymbol{f}(\partial, \alpha) J, \varphi\rangle=\left\langle\mathcal{F}^{-1}\left[\left|f^{\circ}\right|_{p}^{\alpha} \mathcal{F}[J]\right], \varphi\right\rangle & =\left\langle J, \mathcal{F}\left[\left|f^{\circ}\right|_{p}^{\alpha} \mathcal{F}^{-1}[\varphi]\right]\right\rangle \\
=\left\langle J, \mathcal{F}\left[\left|f^{\circ}(-\xi)\right|_{p}^{\alpha} \mathcal{F}[\varphi](-\xi)\right]\right\rangle & =\left\langle J, \mathcal{F}^{-1}\left[\left|f^{\circ}(\xi)\right|_{p}^{\alpha} \mathcal{F}[\varphi](\xi)\right]\right\rangle \\
& =\langle J, f(\partial, \alpha) \varphi\rangle .
\end{aligned}
$$

(ii) The formula follows from the fact that $\left|f^{\circ}\right|_{p}^{\alpha} \mathcal{F}[J] \in \Psi^{\prime}\left(\mathbb{Q}_{p}^{4}\right)$ by using Proposition 5

Definition 3. Consider $\boldsymbol{f}(\partial, \alpha): \mathbf{\Phi}\left(\mathbb{Q}_{p}^{4}\right) \rightarrow \boldsymbol{\Phi}\left(\mathbb{Q}_{p}^{4}\right)$, and the equation

$$
\boldsymbol{f}(\partial, \alpha) u=\varphi, \quad \varphi \in \mathbf{\Phi}\left(\mathbb{Q}_{p}^{4}\right) .
$$

A classical solution of (4.3) is a function $u$ belonging to the domain of $\boldsymbol{f}(\partial, \alpha)$ which satisfies the equation. A fundamental solution of (4.3) is a distribution $E_{\alpha} \in$ $\boldsymbol{\Phi}^{\prime}\left(\mathbb{Q}_{p}^{4}\right)$ such that $u(x)=\left(E_{\alpha} * \varphi\right)(x)$ is a classical solution of 4.3) for any $\varphi \in$ $\Phi\left(\mathbb{Q}_{p}^{4}\right)$.

LEMma 13. The following two assertions are equivalent:

(i) $E_{\alpha} \in \boldsymbol{\Phi}^{\prime}\left(\mathbb{Q}_{p}^{4}\right)$ is a fundamental solution of 4.3);

(ii) $\boldsymbol{f}(\partial, \alpha) E_{\alpha}=\delta$ in $\boldsymbol{\Phi}^{\prime}\left(\mathbb{Q}_{p}^{4}\right)$.

Proof. (i) $\Leftrightarrow \boldsymbol{f}(\partial, \alpha)\left(E_{\alpha} * \varphi\right)=\varphi$ for any $\varphi \in \mathbf{\Phi}\left(\mathbb{Q}_{p}^{4}\right) \Leftrightarrow\left|f^{\circ}\right|_{p}^{\alpha} \mathcal{F}\left[E_{\alpha}\right]=1$ in $\Psi^{\prime}\left(\mathbb{Q}_{p}^{4}\right) \Leftrightarrow \boldsymbol{f}(\partial, \alpha) E_{\alpha}=\delta$ in $\boldsymbol{\Phi}^{\prime}\left(\mathbb{Q}_{p}^{4}\right)$.

Theorem 3. (i) The function

$$
E_{\alpha}(x)= \begin{cases}\frac{1-p^{-\alpha}}{1-p^{\alpha-2}}|f(x)|_{p}^{\alpha-2} & \text { if } \quad \alpha \neq 2 \\ -\frac{1-p^{-2}}{\ln p} \ln |f(x)|_{p} & \text { if } \quad \alpha=2\end{cases}
$$

is a fundamental solution of (4.3).

(ii) Consider $|f|_{p}^{s} \in \mathbf{\Phi}^{\prime}\left(\mathbb{Q}_{p}^{4}\right), s \in \mathbb{C}$. Then

$$
\boldsymbol{f}(\partial, 1)|f|_{p}^{s+1}=\frac{\left(1-p^{s+1}\right)\left(1-p^{-s-2}\right)}{\left(1-p^{-s-3}\right)\left(1-p^{s}\right)}|f|_{p}^{s} \text { in } \quad \boldsymbol{\Phi}^{\prime}\left(\mathbb{Q}_{p}^{4}\right)
$$

Here we are identifying $|f|_{p}^{s}$ with its meromorphic continuation.

Proof. (i) By Lemma 13 we have to show the existence of a distribution $E_{\alpha}$ in $\boldsymbol{\Phi}^{\prime}\left(\mathbb{Q}_{p}^{4}\right)$ satisfying $\boldsymbol{f}(\partial, \alpha) E_{\alpha}=\delta$, which is equivalent (by Lemma 12 (ii)) to solve $K_{-\alpha} * E_{\alpha}=\delta$. By Theorem 2 this equation has unique solution $E_{\alpha}=K_{\alpha}$. Finally $u=E_{\alpha} * \varphi=\mathcal{F}^{-1}\left(\frac{\mathcal{F}(\varphi)}{\left|f^{\circ}\right|_{p}^{\alpha}}\right) \in \mathbf{\Phi}\left(\mathbb{Q}_{p}^{4}\right)$ for $\varphi \in \mathbf{\Phi}\left(\mathbb{Q}_{p}^{4}\right)$.

(ii) Note that

$$
|f|_{p}^{s+1}=\frac{1-p^{s+1}}{1-p^{-s-3}} K_{s+3} \text { in } \mathbf{\Phi}^{\prime}\left(\mathbb{Q}_{p}^{4}\right) \text { for } s \notin\left\{-3+\alpha_{k}\right\} \cup\left\{-1+\alpha_{k}\right\} .
$$


Then by Lemma 12 (ii) and Theorem 2

$$
\begin{gathered}
f(\partial, 1)|f|_{p}^{s+1}=K_{-1} *|f|_{p}^{s+1}=\left(\frac{1-p^{s+1}}{1-p^{-s-3}}\right) \\
K_{-1} * K_{s+3}=\left(\frac{1-p^{s+1}}{1-p^{-s-3}}\right) K_{s+2} \\
=\frac{\left(1-p^{s+1}\right)\left(1-p^{-s-2}\right)}{\left(1-p^{-s-3}\right)\left(1-p^{s}\right)}|f|_{p}^{s}
\end{gathered}
$$

in $\boldsymbol{\Phi}^{\prime}\left(\mathbb{Q}_{p}^{4}\right)$ for $s \notin\left\{-1+\alpha_{k}\right\} \cup\left\{-2+\alpha_{k}\right\} \cup\left\{-3+\alpha_{k}\right\} \cup\left\{\alpha_{k}\right\}$. The announced

formula follows by analytic continuation, since the distributions $\boldsymbol{f}(\partial, 1)|f|_{p}^{s+1}$ and $\frac{\left(1-p^{s+1}\right)\left(1-p^{-s-2}\right)}{\left(1-p^{-s-3}\right)\left(1-p^{s}\right)}|f|_{p}^{s}$ agree on an open and connected subset of the complex plane.

REMARK 3. Similar results are valid for pseudodifferential operators attached to elliptic quadratic forms of dimension 2.

\section{References}

[1] S. Albeverio, A.Yu. Khrennikov, V.M. Shelkovich, Theory of $p$-Adic Distributions: Linear and Nonlinear Models, London Math. Soc. Lecture Note Ser., vol. 370, Cambridge University Press, Cambridge, 2010.

[2] V. A. Avetisov, A. Kh. Bikulov, On the ultrametricity of the fluctuation dynamic mobility of protein molecules, Proc. Steklov Inst. Math. 265 (1) (2009) 75-81.

[3] Georges de Rham, Solution élémentaire d'opérateurs différentiels du second ordre, Ann. Inst. Fourier. Grenoble 8 (1958) 337-366.

[4] Gel'fand I. M., Shilov G.E., Generalized Functions, vol 1., Academic Press, New York and London, 1977.

[5] J.-I. Igusa, An introduction to the theory of local zeta functions, AMS/IP Studies in Advanced Mathematics, 2000.

[6] Yoshiyuki Kitaoka, Arithmetic of quadratic forms,1993.

[7] Jun-ichi Igusa, Some results on p-adic complex powers, Amer. J. Math. 106 (5) (1984) 10131032.

[8] A.N. Kochubei, Pseudo-Differential Equations and Stochastics over Non-Archimedian Fields, Pure Appl. Math., vol. 244, Marcel Dekker, New York, 2001.

[9] Johan A. C. Kolk, V. S. Varadarajan, Riesz distributions. Math. Scand. 68 (2) (1991) 273-291.

[10] S. Rallis, G. Schiffmann, Distributions invariantes par le groupe orthogonal. Analyse harmonique sur les groupes de Lie (Sém., Nancy-Strasbourg, 1973-75), pp. 494-642. Lecture Notes in Math., Vol. 497, Springer, Berlin, 1975.

[11] Boris Rubin, Riesz potentials and integral geometry in the space of rectangular matrices. Adv. Math. 205 (2) (2006) 549-598.

[12] Marcel Riesz, L'intégrale de Riemann-Liouville et le problème de Cauchy, Acta Math. 81, (1949) 1-223.

[13] M. Sato, T. Shintani, On zeta functions associated with prehomogeneous vector spaces, Ann. Math. 100 (2) (1974) 131-170.

[14] Fumihiro Sato, $p$-adic Green functions and zeta functions. Comment. Math. Univ. St. Paul. 51 (1) (2002) 79-97.

[15] Fumihiro Sato, On functional equations of zeta distributions. Automorphic forms and geometry of arithmetic varieties, 465-508, Adv. Stud. Pure Math., 15, Academic Press, Boston, MA, 1989.

[16] Fumihiro Sato, Functional equation of the local zeta functions of quadratic forms, manuscript, 2002.

[17] J.J. Rodríguez-Vega, W.A. Zúñiga-Galindo, Taibleson operators, $p$-adic parabolic equations and ultrametric diffusion, Pacific J. Math. 237 (2) (2008) 327-347.

[18] J.J. Rodríguez-Vega, W.A. Zúñiga-Galindo, Elliptic pseudodifferential equations and Sobolev spaces over $p$-adic fields, Pacific J. Math. 246 (2) (2010) 407-420.

[19] M. H. Taibleson, Fourier analysis on local fields, Princeton University Press, 1975. 
[20] J. T. Tate, Fourier analysis in number fields, and Hecke's zeta-functions. 1967 Algebraic Number Theory (Proc. Instructional Conf., Brighton, 1965) pp. 305-347 Thompson, Washington, D.C.

[21] V.S. Vladimirov, I.V. Volovich, E.I. Zelenov, p-Adic Analysis and Mathematical Physics, Ser. Soviet and East European Math., vol. 1, World Scientific, River Edge, NJ, 1994.

[22] André Weil, Sur certains groupes d'opérateurs unitaires, Acta Math. 111 (1964) 143-211.

[23] W. A Zúñiga-Galindo, Igusa's local zeta functions of semiquasihomogeneous polynomials, Trans. Amer. Math. Soc. 353 (8) (2001) 3193-3207.

[24] W. A. Zúñiga-Galindo, Fundamental Solutions of Pseudo-differential Operators over $p$-adic Fields, Rend. Sem. Mat. Univ. Padova 109 (2003) 241-245.

[25] W.A. Zúñiga-Galindo, Parabolic equations and Markov processes over $p$-adic fields, Potential Anal. 28 (2008) $185-200$.

[26] W. A. Zúñiga-Galindo, Local zeta functions and fundamental solutions for pseudo-differential operators over p-adic fields, p-Adic Numbers Ultrametric Anal. Appl. 3 (4) (2011) 344-358.

Universidad Nacional de Colombia, Departamento de Matemáticas, Ciudad UniverSitaria, Bogotá D.C., Colombia.

E-mail address: ofcasass@unal.edu.co

Centro de Investigación y de Estudios Avanzados del Instituto Politécnico Nacional, Departamento de Matemáticas, Unidad Querétaro, Libramiento norponiente \#2000, Fracc. Real de Juriquilla. Santiago de Querétaro, Qro. 76230, México.

E-mail address: wazuniga@math.cinvestav.edu.mx 